\title{
Green synthesis of gold nanoparticles and its diverse applications in various fields
}

Shiva Shirotiya*, Bhanumati Singh, V. S. Chauhan

Department of Biotechnology, Science Block, Bundelkhand University, Jhansi, India.

Received: 9/7/2017; Revised: 13-07-2017; Accepted: 29-07-2017

\begin{abstract}
Nanobiotechnology is a growing field due to its wide variety of applications in many fields of science and technology. Use of Nanoparticles in biomedical and biotechnology is due to its great surface area, improved permeability, retention effect etc. Green synthesized gold nanoparticles are very much useful for different applications in nanobiotechnology because of its properties and ecofriendly nature. Because of its chemical and physical properties, Colloidal gold has different uses in biotechnology. There are many ways to synthesize gold nanoparticles but green synthesis method is more appropriate as compared to others. This review addressed basically on the different methods of synthesis of gold nanoparticles but is also focused on its green synthesis, different shapes, sizes and various applications in each and every field of life. To be concise, AuNPs are potent tool in targeting drug delivery and biomedical application.
\end{abstract}

Keywords: Biosynthesis, Nanoparticle, AuNPs, Drug, Nanotechnology, targeted drug delivery

Abbreviations: AuNP (Gold nanoparticle), GNP (Gold nanoparticle), TOAB (Tetraoctylammonium bromide), $\mathrm{HAuCl}_{4}$ (Gold III Chloride), Ag (Silver), Au (Gold), SERS (Stimuli responsive surface enhanced Raman Scattering), PEG (Polyethylene glycol), NP (Nanoparticles), HeLA (Oldest and commonly used human cell line derived from cervical cancer cells from Henrirtta Lacks, a cancer patient)

\section{Introduction}

Nanotechnology is the newly emerging field of studying the matter on nanoscale range, usually 10-100 nanometers. In recent days, Nanotechnology is considered to be the one of the most active field of research in biological sciences. Nanotechnology is a field that is rising gradually, having an effect altogether over life of human and making a growing sense of excitement in the life sciences particularly biomedical devices and biotechnology. This is clearly proved from various research papers published in nanotechnology field over a period of time. When the size of nanoparticles reduced to nano meter, it showed its colossal potential. The chemical, electrical, mechanical, optical, magnetic and electro-potential properties of NPs differ from those of their bulk materials, which is due to their high surface to volume ratio. Whereas their physiological and biological properties can be modulated according to the desired application (Hajipour J. M. et al., 2012). So, nanoparticles are a link between bulk material, atomic or molecular structures which has a spectacular effect on its properties as compared to non-scale forms from the identical material (Kherde Y. et al., 2014).

Due to diminution, changes within the physical and chemical properties offers the advancement of novel functional characteristics to nanomaterials. Nanoparticles are now-a days identified for their uses in diverse areas like health care, biomedical, tissue engineering, gene delivery, mechanics, drug designing, optics, food industry, environmental, optical devices and many more. The metallic nanoparticles among all the mentioned areas are considered to be more assured for its exceptional antibacterial properties due to their massive area to volume ratio relation which can help in overcoming the microbial resistance against the antibiotics. Being microbial resistance is a global problem, improvement in drug designing and target delivery has diverted attention of researchers to use metallic nanoparticles for the

*Corresponding Author:

Shiva Shirotiya

Research Scholar, Department of Biotechnology

Bundelkhand University Jhansi (UP), India.

E-mail: shiva.shirotiya@gmail.com purpose. The various metallic nanoparticles that have gained tremendous thought in recent times attributable to their imperative significance area unit i.e, silver, gold, platinum, Titanium, palladium, iron, metallic element and copper and then forth (Ahmed S et al., 2016). Attributable to the attention and use of nanoparticles numerous scientists have concentrated on nanoparticles synthesis utilizing different Physico-chemical techniques. These strategies accessible for the various nanoparticles synthesis are reverse micelle, ion sputtering, reduction, hydrothermal, sol gel, etc. Although it is quite expensive and not environment friendly which include utilization of toxic chemicals which are responsible for different biological risks. The procedures utilizing reagents which occurs naturally like extracts from plants, fungus, sugars, microorganism, biodegradable polymers as reductants and stabilizing agents might be regarded as alternative approach to inorganic nanoparticles synthesis. Nanoparticle synthesized from plant extract are better over other various methods as it is quite simple, one step process, cost-effective, eco friendly (Ahmed S and Ikram $S, 2015)$. Amongst of all the diverse metal nanoparticles, nanoparticles from gold is quite imperative due to its use in medicine i.e, in the treatment of arthritis and cancer.

This review focuses on various ways of synthesis of gold nanoparticles especially green synthesis. This article will also elaborate on different shape, sizes and applications of gold nanoparticles in various fields with its future prospects.

\section{Gold Nanoparticle}

Bulk gold is delicate, malleable, transition metal and usually a rare chemically active elements, while frequently used as a valuable metal in jewellery. Gold in bulk is solid yellow and its nature is inert whereas colloidal solution of gold nanoparticles is wine red in color, highly reactive which accounts for its novel applications (Kherde Y. et al., 
2014). GNPs surprisingly contrast from the bulk gold as a result of quantum size constrainment forced by Nano scale regimen. The electronic, catalytic, and magnetic properties of GNPs depend for the most part on their size and shape. Like, circular GNPs demonstrate at wavelength of $\sim 520 \mathrm{~nm}$ a strong absorption band yet it is missing for tiny particles of $\leq 2 \mathrm{~nm}$ and also for bulk gold. With variety of outstanding properties, when GNPs are controlled successfully, it might be connected to a vast range of uses over the area of medicine and science, environment, and technology.

Table 1. Showing applications of gold nanoparticles with different shapes.

\begin{tabular}{|c|c|c|}
\hline Shape & Size & Application \\
\hline Nanorod & $2-5 \mathrm{~nm}$ & $\begin{array}{l}\text { Drug delivery and photothermal } \\
\text { therapy (Guo Q. et al., 2014) }\end{array}$ \\
\hline $\begin{array}{l}\text { Hollow } \\
\text { particles }\end{array}$ & $25 \mathrm{~nm}$ & $\begin{array}{l}\text { Photo-electronics, catalysts and cancer } \\
\text { therapy } \\
\text { (Ganeshkumar M. et al., 2012) }\end{array}$ \\
\hline $\begin{array}{l}\text { Triangular } \\
\text { particles }\end{array}$ & $\begin{array}{l}3.85 \mathrm{~nm}- \\
7.13 \mathrm{~nm}\end{array}$ & $\begin{array}{l}\text { extremely effective against K.pneumonia } \\
\text { and E.coli (Muravala P. et al., 2014) }\end{array}$ \\
\hline Nanocube & $50 \mathrm{~nm}$ & $\begin{array}{l}\text { Field enhancement use and refractive } \\
\text { index sensing (Pissuwan D. et al., } \\
\text { 2011) }\end{array}$ \\
\hline Nanocage & $50 \mathrm{~nm}$ & $\begin{array}{l}\text { Efficient molecular agent for } \\
\text { nonlinear endomicroscopy imaging } \\
\text { (Giljohann D. A. et al., 2010) and in- } \\
\text { vivo medical applications (Bisker G. et } \\
\text { al., 2012). }\end{array}$ \\
\hline Nanobelt & $\begin{array}{l}\text { Thickness, } \\
\sim 80 \mathrm{~nm}, \\
\text { Width, } \\
20 \mu \mathrm{m}, \\
\text { Length, } \sim 0.15 \\
\text { m. }\end{array}$ & Strain sensors \\
\hline
\end{tabular}

Ref: Khan et al., 2014

Figure 1. Showing different shapes of gold nanoparticles.

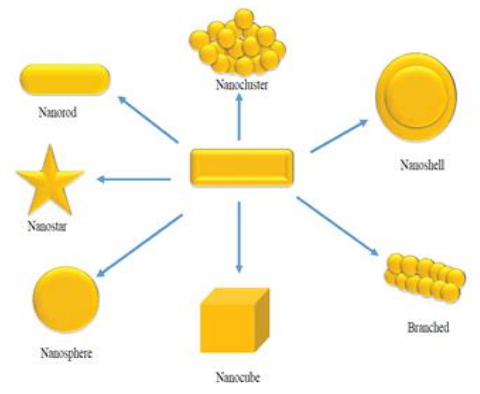

Ref: Alaqad K et al., 2016

Nanoparticles prepared from $\mathrm{Au}$ are used in the field of radiation medicine as a brilliant enhancer and in the treatment of radiation due to its skill in drug delivery. Moreover, Gold Nanoparticles have various utilization in Nanotechnology like proteins marking and detection of biomolecular complexes. Since Au Nanoparticle are nontoxic with extensive surface area and might be altered by means of different molecules, therefore it can be beautifully utilized in the fields of biomedical. Because of its optical properties and compatibility it is used in the field of Biochemistry. Nanoparticles are high-quality curative agents because of its ease transport in diseased cell and in carrier loading drug (Alaqad K and Saleh T.A., 2016).

\section{Sizes of Gold nanoparticles}

Gold nanoparticles would be classified depending upon on the size, shape and physical properties. Gold nanoparticles indicates distinctive sizes varies from $1-8$ $\mu \mathrm{m}$ and particles likewise exhibit diverse shapes, for example, sphere, nanorods, nanoshell, nanocages, branched, nanocluster and nanostar (Das M et al., 2011). The essential achievement in the field of AuNPs was gold nanospheres, regardless of the way that they were not precisely spherical (Das M et al., 2011). Later on various different structures were also obtained which is shown in Fig.1 with their applications in diverse fields (Table No.1). Other types of gold nanoparticles were also created with SERS. The synthesis techniques for nanomaterials were continuously developing for long periods. Subsequently, the various synthetic protocols become accessible, and their shapes and sizes could be controlled and are innovative, for example nanocages. From all the different shapes (nanospheres, nanaorods, nanoshells, nanaocages and SERS NPs) nanoparticles having triangular shape demonstrated alluring optical properties when compared with the nanoparticles of spherical shape.

\section{Au Nanospheres}

Gold colloids is very well known by the other name called "gold Nanospheres". The width range from 2 to $100 \mathrm{~nm}$ which would be synthesized by reducing aqueous $\mathrm{HAuCL}_{4}$ solution with addition of different reducing agents under different conditions and parameters, where most generally used reducing agent was the citrate, developed mono disperse gold nanospheres (Das M et al., 2011, Frans G.,1973, Turkevich J et al., 1951). Lesser the citrate amount large amount of nanospheres would yield. Nanospheres size could be controlled by shifting the concentration of gold and citrate. Significant obstacle in above procedure is for lesser yield of gold Nanospheres and the limited use of water, as solvent (Das M et al., 2011).

\section{Au nanorods}

Various methods for synthesizing Au Nanorods were used. They are synthesized by template strategy, depending on the electrochemical deposits on Gold inside the pores of alumina template membrane or nanoporous polycarbonate. The diameter of gold nanorods could be pre-chosen by means of knowing the diameter of the template membrane pores. Their length can be controlled by the amount of Au deposited inside the membrane pores. Whereas, the main limitation with the technique was low yield of Gold nanorods, due to formation of single layer of nanorod. The most commonly used method of gold nanorod synthesis could be Seed Mediated Synthesis resulting highest yield ratio in comparison with Turkevich and Burst methods (Jana N. R. et al., 2001, Busbee B. D. et al., 2003)

\section{Au nanoshell}

Nanoshell alluded as a kind for round nanoparticle with a dielectric core, which might have been secured by thin metallic shell (Like Au). Such nanoshells included quasi particle, called plasmon, produced from quantum plasma oscillation where electrons simultaneously oscillate with respect to all ions. Due to high reflective optical and compound properties of the nanoshells, its real requisition might have been for biomedical optical imaging, fluorescence enhancement of weak molecular emitters, therapeutic applications, surface enhanced Raman spectroscopy and surface enhanced infrared Absorption spectroscopy (Brinson B. L. et al., 2008).

\section{Au Nanocages}

$\mathrm{Au}$ nanocages, comprised controllable pores on its surface, were synthesized by galvanic replacement reaction of truncated silver nanocubes and aqueous 
$\mathrm{HAuCl}_{4}$. Furthermore, it might have been observed that morphology of the silver nanostructures can be controlled through reduction of Pylol. Here, $\mathrm{AgNO}_{3}$ has been reduced by ethylene glycol leading to production of silver atoms, which on reduction yielded seeds or nanocrystals. Required nanostructures were processed through additional silver atoms and furthermore at the same time structures of silver seed crystalline can bb controlled with polyvinylpyrrolidone addition, which posed a selective binding to the surface (Chen J. et al., 2006). Gold nanocages might provide major advantages e.g., (i) their surface Plasmon resonance peaks might make tuned by changing the proportion of the Gold nanocubes and $\mathrm{HAuCl}_{4}$. Covering spherical region between $500 \mathrm{~nm}$ to $1200 \mathrm{~nm}$; (ii) Absorption coefficient might vary by controlling void size and number of truncated corners; (iii) those Gold nanocages might show resonance peaks in the near Infra Red region with size of $<50 \mathrm{~nm}$; Also (iv) surface modulations can be performed and applied in various biomedical applications (Chen J. et al., 2005).

SERS (Stimuli-responsive Surface enhanced Raman Scattering) NPs

Optical technique SERS has more advantages as compared to general traditional technologies. The significant advantage of Stimuli-responsive Surface Enhanced Raman Scattering might be the chemiluminescence and fluorescence, high levels of multiplexing, higher sensitivity, greater performance in blood, robustness and other biological matrices. $60 \mathrm{~nm}$ modified gold nanospheres with Raman reporter were stabilized by a process of a layer of thiolated polyethylene glycol. One more kind of SERS nanoparticle was formed by a molecular layer of Raman-active molecular layer on silica coated Au core. It ensures the inertness and physical robustness to different conditions of environment and by silica chemistry simplified the modification of surface. Thiol groups Conjugation were introduced onto the shell of silica, and the PEG chains activated by maleimide improved biocompatibility (Cai W. et al., 2008).

\section{Gold Nanoparticle Synthesis}

Gold Nanoparticle can be synthesized by two ways, either from "bottom to top or "top to bottom" method. In bottom to top method nanoparticles can be synthesized by chemical reduction and by the use of bacteria, fungi, plants, etc. (Biological approach) by self assembling of atoms to newer nuclei which develop into a particle of nanoscale (Fig. 2a) whereas in top to bottom method, substances break down into finer particles by reduction of size with different lithographic techniques for example sputtering, grinding, laser ablation, milling, etc. But both these methods used for nanoparticles synthesis are harmful due to use of harmful chemicals which are responsible for different biological risks and are quite costly (Fig. 2b). Different methods can be used for synthesis of gold nanoparticles. The techniques used for nanoparticles preparation can be biological, physical and chemical (Ahmed S et al., 2016).

\section{Chemical methods}

In chemical techniques gold nanoparticles mostly produced by reduction of hydrochloroauric acid $\left(\mathrm{HAuCl}_{4}\right)$, using some kind of stabilizing agent. After dissolving $\mathrm{HAuCl}_{4}$, quickly stirred the solution while mixing a reducing agent leading to the reduction of $\mathrm{Au}^{+3}$ ions to neutral gold ions (Sharma C. K. et al., 2015).
Figure 2. Showing Bottom to top approach for synthesis of AuNPs (a) and top to bottom bottom approach for synthesis of AuNPs (b)
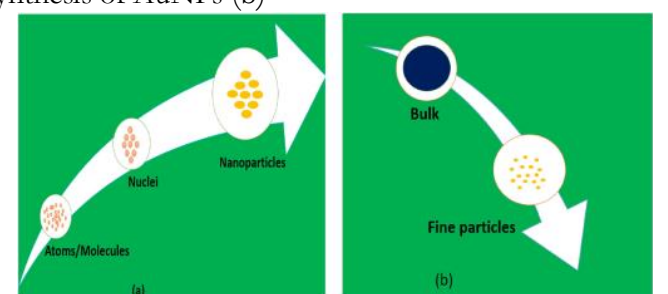

Ref: Ahmed S et al., 2016

\section{Turkevich method}

The turkevich method is the most widely used method for spherical gold nanoparticles synthesis of size ranging from 10-20 nm. It involves reaction of fine amounts of hot $\mathrm{HAuCl}_{4}$ in by adding reducing agents like ascorbic acid, citrate, amino acids, or Ultra Violet light leading to the formation of colloidal gold due to citrate ions which act as a capping agent and reducing agent (Ahmed $\mathrm{S}$ et al., 2014). To deliver larger particles, sodium citrate concentration should be less, down to $0.05 \%$, afterwards simply would not able to reduce entire gold. The reduction in the amount of sodium citrate will reduce citrate ions in solution which is accessible for particles stabilization. Therefore it will lead to the particles to aggregate into larger, unless the whole surface area of all the particles becomes small enough to be cover by the citrate ions exist in the solution (Sharma C. K. et al., 2015).

\section{Brust Method}

Burst method is a two stage to produce 1.5-5.2 $\mathrm{nm} \mathrm{Au}$ Nanoparticles by means of natural solvents and by changing the ratio of thiol to $\mathrm{Au}$. This technique was reused from Faraday's two stage procedure. This technique includes gold salt exchange from aqueous solution for a natural organic liquids (Like toluene) which is used as phase transfer catalyst and the stabilizing agent (like TOAB solution in toulene). The Au is then reduced using sodium borohydride in presence of an Alkanethiol. It stabilizes the Au Nanoparticles, bringing about change in the colour of the reaction to dark brown from orange. Purification of Au Nanoparticles from TOAB stabilized with dodecanethiol was accounted by Schriffin (Ahmed S et al., 2014).

\section{Seeded growth method}

In Brust and Turkevich strategies, spherical $\mathrm{Au}$ Nanoparticles are formed, however in Seeded development technique $\mathrm{Au}$ Nanoparticles can formed in variety of Nanostructures, like tubes, cubes, rods,. Seed mediated growth is the widely used approach to synthesized AuNPs in different shapes (Zhi- Chuax X. et al., 2007). Firstly seed particles synthesized by reduction of gold salts by the use of reducing agents for example sodium borohydride. In presence of a weak reducing agent like ascorbic acid, seed particles are then added to metal salt solution and guiding agent to structure. This will avoid advance nucleation and speed up the anisotropic development of Au Nanoparticle. AuNPs Geometry could be adjusted by structure coordinating agents, reducing agents, and by differing seeds concentration (Sharma C. K. et al., 2015).

\section{Physical Methods}

$\gamma$-light strategy was ended up being best for AuNPs synthesis with convenient size and high virtue. The 
Gamma irradiation technique is used for synthesis of AuNPS measuring 2 to $40 \mathrm{~nm}$. This strategy utilized solution of regular polysaccharide alginate as stabilizer. Akhavan A et al., gave single step $\gamma$-light strategy to integrated AuNPS of size $2 \mathrm{~nm}-7 \mathrm{~nm}$ by utilizing cowlike egg serum white protein as stabilizer. Likewise, other used physical system which can be used are ultrasonic waves, microwaves, laser ablation, solvothermal method, electrochemical and photochemical decrease is accessible in literature for AuNPs synthesis (Ahmed S et al., 2014).

Gold Nanoparticles Biosynthesis: Environment Friendly and a Green Approach

From last few years, nanoparticles from gold has been utilized in many ways for example, targeted drug making, cosmetics, biosensors, and in various applications of biomedical. $\mathrm{Au}$ nanoparticles are potential agents for antimicrobial activity showing bactericidal and fungicidal activity against different microbes. Since Au nanoparticles are stable, it promised to be a building step in the new technology in future. Au nanoparticles can be produced by utilizing a large number of physical and chemical procedures. Though, these methodology is quite costly and have ecological risks associated with their manufacture. Decreasing the utilization of chemicals which are toxic utilized as a part of typical AuNPs synthesis, researchers are effectively focusing on alternative techniques for synthesis utilizing organic materials (proteins, polysaccharides, polyphenol, and so on) also known as green synthesis of gold nanoparticles (Ahmed S et al., 2016).

These green $\mathrm{Au}$ nanoparticles synthesis is getting predominant because of its salts get reduced at room temperature, cost-effective, non-harmful and eco-friendly. That straight forward, one step strategy is suitable for production at large scale as its cheep, fast, and eco friendly therefore safe for using in clinical research. Its biosynthesis is growing as a point in the field of biotechnology and nanotechnology because of a rising need to synthesize environment friendly methods. The carbonyl and hydroxyl functional group of biomolecules could decrease $\mathrm{Au}^{3+}$ particles to $\mathrm{Au}^{0}$. $\mathrm{Au}^{0}$ finally capped to frame stabilized Gold NPs. By this strategy the issue of reactants biosafety has been utilized for the AuNPs synthesis (Ahmed S et al., 2016). Nagajyothi et al., (2014) utilized extract of Lonicera japonica flower for synthesis of $\mathrm{Au}$ nanoparticles. Amino Acids, Amide, alcohols and alkane introduce in these extracts are required in the bioreduction and stabilization of $\mathrm{Au}$ nanoparticles. Combination of nanoparticles indicated Antimicrobial action against Gram +ve (Bacillus subtilis and) Staphylococcus aureus microscopic organisms, Gram-ve (E.coli) microscopic organisms, and fungus (C. albicans and $S$. cerevisiae) (Nagajyothi P. C. et al., 2014). Aqueous leaves extract of Azadiracbta indica is utilized for gold nanoparticles synthesis showing tough cytotoxic impacts against MDA-MB-231 cells, proposing that naturally incorporated Au nanoparticles may utilized as best anticancerous agents for breast tumor treatment (Krishnaraj C. et al., 2014). Gold nanoparticles biosynthesis by E. coli $\mathrm{DH} 5 \alpha$ for its use on coordinate electrochemistry of haemoglobin has been reported. On the surface of microorganism, nanoparticles bounds and this combination might utilized for function in understanding the immediate haemoglobin electrochemistry (Du L. et al., 2007). A fast development of gold nanoparticles in short duration have been accomplished utilizing Sargassum wightii (Sharma C. K. et al., 2015). TEM demonstrates development of very much scattered gold nanoparticle with molecule size ranges from 8-12 nm (Singaravelu G. et al., 2007). Extract of Cerasus serrulata leaves has been utilized to biosynthesize Au nanoparticles of spherical shaped with size approx. 5 $\mathrm{nm}-25 \mathrm{~nm}$ having antibacterial activity against Escherichia coli and against S. aureus bacteria (Karthik R. et al., 2016). The beauty of using plant extract is that there is no requirement to include any stabilising agents, since phytochemical itself is utilized as a reducing and stabilizing agents.

\section{Gold nanoparticles biosynthesis Mechanism}

Synthesis of $\mathrm{Au}$ nanoparticles mechanism by using various biological agents is yet unknown. Various chemical entities acts as reducing agents which are present in biogenic compounds thus reacts with metal ions leading to its reduction and thereby metal nanoparticles synthesis (Fig.3) (Hulkoli N and Taeanath T. C., 2014).

Figure 3. Showing synthesis of AuNPs with the use of reductants present in biogenic compounds.

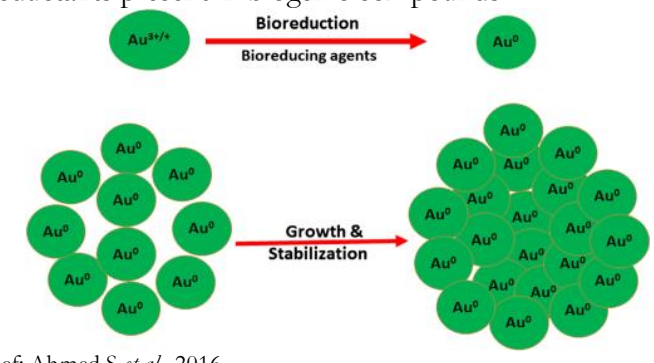

Ref: Ahmed S et al., 2016

\section{Applications of AuNPs}

AuNPs have exceptional magnetic and electric properties because of their size and shape that's why they have acquired attention in research field especially in the field of biological and chemical sensing, photo thermal therapy, biological tagging, optoelectronics, DNA labelling biomedical imaging, etc (Fig. 4) (Alanazi F. K. et al., 2010).

Figure 4. Showing applications of AuNPs in various fields

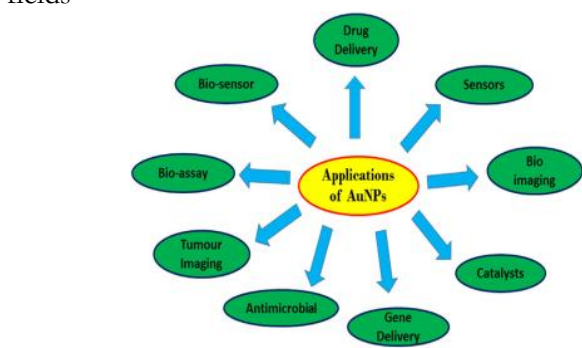

Ref: Ahmed S et al., 2016

\section{Biosensors}

Metal NPs are most likely used in various natural and electrochemical detecting framework because of its chemical and physical property. NPs coated with biological or molecular materials, for example, antibodies and collagen, can be used as bioinorganic interface. By controlling size of NPs, efficient and effective fluorescent probes can be made that discharge fine brightened illumination in broad array of wavelength. DNA and protein can be immobilized on surface of NPs after that intensity and wavelength of NPs are monitored and can 
be used as a DNA and protein biosensor respectively (Sharma C. K. et al., 2015). Sensors based on Au nanoparticles can identify different metal ion by working on color change principle due to AuNPs accumulation. These sensors have been generally utilized for identification of copper, mercury, lead, and arsenic in water (Khan A. K. et al., 2014).

\section{Immunoassay}

Different immuno assay have been planned utilizing Gold Nanoparticles functionalized with various antibodies for example, human Immunoglobin $\mathrm{G}$ and antibodies against harmful microbes. Immunosensors have been recently created utilizing single chain fragment variable antibodies rather than traditional mono or polyclonal antibodies ( Di Pasqua A. J. et al., 2009, Peng Z. et al., 2007).

\section{Disease diagnostics}

An ordinary treatment for cancer which incorporates radiation, chemotherapy, surgery and hormone treatment have a few limitation as they nonspecifically influences the normal and unhealthy cell in human body. NPs can beat the limitation of conventional treatment as it is utilized as drug delivery agent which target the drug in more amount to the tumor site hence improvises cancer treatment. AuNPs can be used in the assembly of electrochemical and amperometeric biosensors which can be used as a detector for tumor particular biomarker (like alpha-fetoprotein) and improves imaging capacity which analyze cancer. In the mid 1980s first clinical trial of NPs for anti-cancer drug delivery was done and in 1995 NPs for the first time had entered in pharmaceutical market. NPs are designed as $\mathrm{pH}$ and temperature sensitive so that it can discharge drug inside more acidic condition consequently acts as potential instrument for malignancy treatment (Pekamwar S.S. et al., 2015).

\section{Preclinical endoscopy imaging}

Despite the fact that improved Raman Spectroscopy is an effective and useful strategy for clinical imaging, the test of restricted light penetration in inner tissues can't be neglected. Hence, in recent report authors proposed for utilizing Raman Spectroscopy for gathering ex-vivo or invivo data by Raman spectroscopy coupling with endoscopy. That approach possibly addresses the test in Raman imaging of limited light penetration (Pekamwar S.S. et al., 2015).

\section{Enzyme immobilization}

For enzyme, AuNPs are being utilized as an immobilization matrices. Gold nanoparticles with carboxyl ended group of thiol were functionalized through conjugation with the enzyme glucose oxidase. Immobilized protein when observed was thermally more stable when compared with free enzymes. These immobilized systems could be exceptionally helpful for a few biotechnological activities in environment and food fields. Hollow $\mathrm{Au}$ nanoshells trapping horse radish peroxides has been synthesized for Identification of small molecules which might enter in the nanoshell (Kumar R. et al., 2005). This strategy enables the compound to stay dynamically active in nanoshell, making it helpful for different biotechnological applications.

\section{Targeted drug and biomolecule delivery}

Functionalized gold nanoparticles has been utilized to target drugs and biomolecules to particular organelles and cell type, for example, mitochondria or nucleus. AuNPs functionalized with 3-mercaptopropinoic acid and PEG were appeared to enter HeLA cells nucleus without any extreme cytotoxicity and consequently could be utilized as nuclear delivery drug carrier (Gu Y. J. et al., 2009). NPs specially nanospheres and nanocapsules into which drug is liquefied, ingested and detached into the surrounding substance plus drug is bounded to a fluid secured by a shell like wall respectively. NPs can overcome the drawback like short circulation prompts to frequent administration and undesired targeting related with the small therapeutic agents. Polymer NPs can broaden the in vivo circulation time from minutes to hours (exceedingly stable); with leaky blood vessels this expands the passive delivery of drug to tissue (control drug discharge from lattice) and its feasibility of administration through different courses that is inhalation and oral administration. Gene delivery by means of NPs can replace defective or missing gene which can cause destruction of defective cell and make impaired cells to return to their normalcy (Pekamwar S.S. et al., 2015).

\section{Improving Air and water quality}

One of the most promising use of AuNPs is improving air and water quality, $\mathrm{CO}$ is a odorless and colorless gas which is very harmful to humans. AuNPs provides an easy solution. AuNPs allows oxidation of carbon monoxide to carbon dioxide that turns an acutely poisonous gas to less toxic one. During recent years it is seen a sharp increase in the use of noble metal nanoparticles for detection of contaminant and water purification. AuNPs has also been used as a efficient adsorbents for removal of significant levels of $\mathrm{Hg}$ to make water potable (Pekamwar S.S. et al., 2015).

\section{Microbes Detection}

Microbial detection can be achieved by various microbiological, molecular and biochemical methods. In one study, oligonucleotides complementary to the unique sequences of heat shock protein i.e,HSP 70 of Cryptosporidium parvum were utilized to functionalize AuNPs, which can be utilize to detect oocytes of cryptosporidium in a colorimetric assay, showing a simple and best method for molecular detection (Javier D. J. et al., 2009).

\section{Future perspectives}

It is an emerging and new area of research in the field of science, where gradually developments are distinguished in warranting a brilliant future for this field. As the nano revolution unfolds, it is essential to develop nano-nature connections between nanotechnology and green domains of the nature. The power of phytochemicals, which commences various chemical transformations within biological system is also well known. Antimicrobial studies of versatile and magical nanoparticles on microbes might open a gate for a innovative range of antimicrobial agents with potent efficacy as compared to available drugs. Moreover, in a vast majority of existing studies, the effect of nanoparticles on micro-organisms is not well known which is now getting considerable attention. Therefore, promoting nanoparticles biosynthesis could influence the commercial use of such nanoparticles in the pharmaceuticals field and other medical sciences which are factors for synthesized nanoparticles via conventional methods. There are certain issues to be dealt with. Several studies still need to be performed to understand the effect of light, temperature, time and several other factors on the formation of gold nanoparticles still requires 
optimization and also on the control of size and shape of the nanoparticles. Furthermore lack of knowledge of chemical components responsible and mechanism for the reduction and stabilization of biosynthesized gold nanoparticles are still challenges for researchers. Therefore, a systematic study is recommended to focus on mechanism of synthesis of gold nanoparticles and effect on size and shape of gold nanoparticles for different applications. The future of nanomedicine lies in multifunctional nanoplatforms which combined both therapeutic components and molecular imaging. Nanoparticle based agents can allow us for efficient, specific or targeted in vivo drug delivery without any kind of systemic toxicity, and the dose delivery. The therapeutic accuracy and efficacy can be measured noninvasively over time is ultimate goal.

\section{References}

1. Ahmed $\mathrm{S}$ and Ikram S. Synthesis of gold nanoparticles using plant extract: An overview. Nano Res. and App. (2015). 1:2471-9838.

2. Ahmed S, Annu, Ikram S and Yudha SS. Biosynthesis of gold nanoparticles: A green approach. J Photochem. Photobio., B: Biology. (2016). 161:141-153.

3. Alanazi FK, Radwan AA and Alsarra IA. Biopharmaceutical applications of nanogold. Saudi Pharm J. (2010). 18: 179-193.

4. Alaqad K and Saleh TA. Gold and silver nanoparticles : Synthesis methods, characterization routes and applications towards drugs. J Environ. Anal. Toxicol. (2016). 6:4 2161-0525.

5. Bisker G, Yeheskely-Hayon D, Minai L and Yelin D. Controlled release of Rituximab from gold nanoparticles for phototherapy of malignant cells. J Control Release. (2012). 162: 303-309.

6. Brinson BE. Nanoshells made easy: improving Au layer growth on nanoparticle surfaces. Langmuir. (2008) 24: 14166-14171.

7. Busbee BD, Obare SO and Murphy CJ. An improved synthesis of high aspect ratio gold nanorods. Adv. Material. (2003) 15: 414-416.

8. Cai W, Gao T, Hong $\mathrm{H}$ and Sun J. Application of Au nanoparticles in cancer nanotechnology. Nanotech. Sci. Appl. (2008). 1: 17-32.

9. Chen J, Saeki F, Wiley BJ, Cang H, Cobb MJ, Zhi-Yuan L, Au L, Zhang H, Kimmey MB and Lix Xia Y. Gold Nanocages: Boconjucation and their potential use as a optical imaging contracts agents. Nano Letters. (2005). 5:473-477.

10. Chen J, Mclellan JM, Siekkinen A, Xiong Y, Li ZY and XIA Y. Facile synthesis of gold-silver nanocages with controllable pores on the surface. J Am. Chem. Soc. (2006). 128:14776-14777.

11. Das M, Shim K H, An S S A and Yi D K.. Review on Gold Nanoparticles and Their Applications. Toxicol. Environ. Health Sci. (2011). 3:193-205.

12. Di Pasqua AJ, Mishler IIRE, Ship YL, Dabrowiak JC and Asefa T. Preparation of antibody-conjugated gold nanoparticles. Material Lett. (2009). 63: 1876-1879.

13. Du L, Jiang $\mathrm{H}$, Liu $\mathrm{X}$ and Wang $\mathrm{E}$. Biosynthesis of gold nanoparticles assisted by Escherichia coli DH5 $\alpha$ and its application on direct electrochemistry of hemoglobin. Electrochem. Commun. (2007). 9: 11651170.

14. Frens $G$. Controlled nucleation for the regulation of the particle size in monodisperse Au suspensions. Nature. (1973). 241: 20-22

15. Ganeshkumar M, Sastry TP, Sathish Kumar M, Dinesh MG, Kannappan S and Suguna L. Sunlight mediated synthesis of gold nanoparticles as carrier for 6- mercaptopurine: Preparation, characterization and toxicity studies in zebrafish embryo model. Mat. Res. Bull. (2012). 47: 2113-2119.

16. Giljohann DA, Seferos DS, Daniel WL, Massich MD, Patel PC and Mirkin CA. Gold nanoparticles for biology and medicine. Angewandte Chem. (2010). 49: 3280-3294.

17. Guo Q, Guo Q, Yuan Jand Zeng J. Biosynthesis of gold nanoparticles using a kind of flavonol: Dihydromyricetin. Colloids and Surfaces A: Physicochem. and Eng. Aspects. (2014). 441: 127-132.

18. Gu YJ, Cheng J, Lin CC, Lam YW, Cheng SH and Wong WT. Nuclear penetration of surface functionalized gold nanoparticles.(2009). Toxicol. Appl. Pharmacol., 237:196-204.

Source of support: Nil;
19. Hajipour JM, Fromm KM, Ashkarran AA, de Aberasturi DJ, de Larramendi IR, Rojo T, Serpooshan V, Parak WJ and Mahmoudi M. Antibacterial properties of nanoparticles. Trend Biotechnol. (2012). 30: 499-511.

20. Hulkoli $\mathrm{N}$ and Taeanath TC. Biosynthesis of nanoparticles using microbes- a review. Colloids Surf B Biointerfaces. (2014). 121:474-483.

21. Jana NR, Gearheart L and Murphy CJ. Seed mediated growth approach for shape controlled synthesis of spheroidal and rod like gold nanoparticles using a surfactant template. Adv. Material. (2001). 13: 13891393 .

22. Javier DJ, Castellanos-Gonzolez A, Weigum SE, White AC and RichardsKortum R Oligonucleotides-gold nanoparticles network for detection of cyptosporidium parnum heat shock protein 70 mRNA. J Clin Microbiol. (2009). 47:4060-4066.

23. Karthik R, Chen S-M, Elangovan A, Muthukrishnan P, Shanmugam R and Lou B-S. Phyto mediated biogenic synthesis of gold nanoparticles using Cerasus serrulata and its utility in detecting hydrazine, microbial activity and DFT studies. J. Colloid Interface Sci. (2016). $468: 163-175$.

24. Khan AK, Rashid R, Murtaza G and Zahra A. Gold Nanoparticles: Synthesis and Applications in Drug Delivery Khan. Tropical J. of Pharma. Research. (2014). 13:1169-1177.

25. Kherde Y, Aguilar ZP, Rodgres H and Hamilton W. Gold nanoparticles: various methods of synthesis and antibacterial applications. Frontiers in Biosci. (2014). 19:1320-1344.

26. Krishnaraj C, Muthukumaran P, Ramachandran R and Balakumaran MD Acalypha indica Linn : biogenic synthesis of silver and gold nanoparticles and their cytotoxic effects against MDA-MB-231, human breast cance cells. Biotechnol. Rep. (2014). $4: 42-49$

27. Kumar R, Maitra AN, Patanjali PK and Sharma P. Hollow gold nanoparticles encapsulating Horseradish peroxide. Biomaterials. (2005). 26:6743-6753.

28. Murawala P, Tirmale A, Shiras A and Prasad BLV. In situ synthesized BSA capped gold nanoparticles: Effective carrier of anticancer drug Methotrexate to MCF-7 breast cancer cells. Mat. Sci. Engineer C. (2014). 34: 158-167.

29. Nagaiyothi PC, Lee KD and Sreekanth TVM. Biogenic synthesis of gold nanoparticles (quasi-spherical, triangle and hexagonal) using Lonicer japonica flower extract and its antimicrobial activity. Synth. React. Inorganic japonica flower extract and its antimicrobial activity.

30. Pekamwar SS, Deshmukh VS and Kalyankar TM.Gold Nanoparticles in biomedical applications. Int. Res. J. Pharm. (2015). 6(10):2230-8407.

31. Peng Z, Chen Z, Jiang J, Zhang X, Shen G and Yu R. A noble immunoassay based on the bissociation of immunocomplex and fluorescence quenching by gold nanoparticles. Ann Chim. Acta. (2007) 583: 40-47.

32. Pissuwan $\mathrm{D}$, Niidome $\mathrm{T}$ and Cortie MB. The forthcoming applications of gold nanoparticles in drug and gene delivery systems. J Control Release. (2011). 149: 65-71.

33. Sharma CK, Sharma M, Verma O and Sharma V. Green synthesis of different nanoparticles and their potential applications in different fields A critical review. Int J Pharm Bio Sci. (2015). 6(3): 555-567.

34. Singaravelu G, Arockiamary JS, Kumar VG and Govindaraju K.. A nove extracellular synthesis of monodisperse gold nanoparticles using marine alga, Sargassum wightii Greville. Colloids Surf. B Biointerfaces (2007). 57:97-101.

35. Turkevich J, Stevenson PC and Hillier J. The nucleation and growth processes in the synthesis of colloidal Au. Discuss Faraday Soc. (1951). 11: $55-75$.

36. Zhi-Chuan X, Cheng-Min S, Cong-Wen X, Tian-Zhong Y, Huai-Ruo Z, Jian-Qi L, Hu-Lin L and Hong-Jun G. Wet chemical synthesis of gold nanoparticles using silver seeds: A shape control from nanorods to hollow spherical nanoparticles. Nanotechnol. (2007). 18:115608.

\section{Cite this article as:}

Green synthesis of gold nanoparticles and its diverse applications in various fields Shiva Shirotiya, Bhanumati Singh, V. S. Chauhan. Annals of Plant Sciences 6.11 (2017) pp. 17201725.

http://dx.doi.org/10.21746/aps.2017.6.11.0

Conflict of interest: Nil. 